\section{Partizipative Technologiepolitik in Österreich?}

\author{
Bericht zu den Projekten \\ „Techpol 2.0“ und „FSA-Energie“
}

\author{
von Michael Nentwich, ITA Wien
}

Im Herbst 2006 schloss das Institut für Technikfolgen-Abschätzung (ITA) eine Studie für den österreichischen „Rat für Forschung und Technologieentwicklung“" (RFT) ab, die zwei wichtige Anliegen der österreichischen Technologiepolitik verknüpft: zum einen mehr „Awareness“ für technologiepolitische Themen in der Bevölkerung zu schaffen und dies mittels Beteiligungsverfahren zu versuchen; zum anderen - über die Bewusstseinsbildung hinaus - das international bewährte Potenzial von partizipativen Verfahren zur Legitimitätssteigerung und zur Stärkung der ,sozialen Robustheit", also der gesellschaftlichen Verankerung von technologiepolitischen Entscheidungen zu nutzen. Seit Sommer 2007 hat das ITA die Gelegenheit, im Auftrag des RFT und zweier Bundesministerien (Innovation/Technologie und Wirtschaft) diese Ergebnisse umzusetzen. Kurz vor Erscheinen dieses Berichtes hat eine partizipative Veranstaltung zum Thema ,Energie und EndverbraucherInnen" stattgefunden.

\section{Techpol 2.0: Awareness - Partizipation - Legitimität}

Im Rahmen der Forschungs- und Technologiepolitik haben wir es heute häufig mit Wertekonflikten zu tun, die die Frage betreffen, welches Wissen wir zu welchen (ethischen) Kosten wollen bzw. wie wir mit diesem umgehen wollen. Diese Wertekonflikte, die in gewissem Ausmaß auch Wissens- und Interessenskonflikte sind, können durch die Abhaltung strukturierter Dialogverfahren unter Einbeziehung von bis dahin nicht involvierten BürgerInnen (Laien) neben Fachleuten und InteressenvertreterInnen wenn schon nicht gelöst, so doch transparenter, umfassender und strukturierter verhandelt werden. Damit besteht sowohl Aussicht auf bessere Entscheidungen, da sie auf breiterer Basis Wissen, Interessen und Werthaltungen einbeziehen, als auch auf die größere Legitimierung von Entscheidungen. Die vorgeschlagenen Verfahren treten neben die bewährten Entscheidungsprozesse, erweitern diese, sind jedoch nicht als Ersatz für sie gedacht.

Die internationale Erfahrung lehrt einerseits, dass es für unterschiedliche Themen und Ziele höchst unterschiedliche Verfahren gibt; das bekannte Verfahren der Konsens- bzw. BürgerInnenkonferenz ist nur eines von vielen möglichen. Andererseits eignet sich auch nicht jedes Thema zu jedem Zeitpunkt für die Behandlung in einem zumeist aufwändig vorzubereitenden öffentlichen Beteiligungsverfahren. Die ITAStudie (Nentwich et al. 2006) stellt daher neun typische und bewährte Verfahren vor, analysiert deren potenziellen Einsatzbereiche und zeigt Wege auf, wie das bestgeeignete Verfahren für ein gegebenes Thema gefunden werden kann. ${ }^{1}$ Ein „Zwischenergebnis“" war die deutsche Übersetzung des flämischen Handbuchs partizipativer Verfahren (Steyaert et al. 2006).

Daraus abgeleitet und auf Basis internationaler Erfahrungswerte wurde in einem nächsten Schritt ein Kriterienkatalog erstellt, mit dessen Hilfe mögliche technologiepolitische Themen daraufhin untersucht werden, ob sie zu einem bestimmten Zeitpunkt in einem partizipativen Verfahren mit Aussicht auf Erfolg verhandelt werden können. Wichtige Kriterien sind dabei insbesondere das potenzielle Interesse der Medien und die politische Konjunktur des Gegenstandes. Außerdem spielen die Komplexität der Materie sowie deren bisherige politische Thematisierung eine wichtige Rolle.

Das ITA hat im Rahmen dieses Projekts testweise zwölf Themen, die bereits auf dem „Radar“ der Technologiepolitik aufgetaucht sind, anhand dieser Gesichtspunkte analysiert und unter Einbeziehung der Ergebnisse von Interviews mit AkteurInnen und ExpertInnen der österreichischen Technologiepolitik eine Auswahl von drei Themen getroffen. Auf Basis eines speziell entwickelten Rasters ${ }^{2}$ war es möglich, jenen Verfahrenstyp zuzuordnen, der am besten für eine Fragestellung passt. Die folgenden drei Themen erschienen im Herbst 2006 für das Abhalten einer partizipativen Veranstaltung im Jahr 2007 besonders gut geeignet:

- eine BürgerInnenkonferenz zur Präimplantationsdiagnostik, 
- $\quad$ eine Citizen Jury zu RFID-Chips (Radio Frequency Identification),

- eine Future Search Conference zum Thema Energieeffizienz. $^{3}$

Auf Basis des von uns erarbeiteten Kriterienkatalogs für die Auswahl von geeigneten Themen und der Systematisierung der bewährten Verfahrenstypen empfahlen wir außerdem, in $\mathrm{Zu}-$ kunft regelmäßig technologiepolitische Entscheidungen transparenter, öffentlichkeitswirksamer (und damit legitmitätserhöhend) zu treffen. Das Screening und die Analyse der aktuellen Themen machen deutlich, dass es eine Vielzahl von gut geeigneten Themen gibt und geben wird, deren Behandlung in partizipativer Art ein gewinnbringender Input für traditionelle Entscheidungsverfahren wäre.

Unsere Analyse des institutionellen Rahmens und der wesentlichen Akteure in Österreich war darauf gerichtet, die potenziellen Adressaten und Auftraggeber für die Einspeisung dieses für Österreich neuartigen technologiepolitischen Inputs aufzuzeigen. Im Zentrum stehen der RFT, die Forschungsförderungsgesellschaft (FFG) und die jeweils relevanten Bundesministerien. Daher traten wir im Herbst 2006 mit dem RFT in Verhandlungen ein, um die Ergebnisse des Projekts Techpol 2.0 umsetzen zu können. Die Entscheidung fiel für das dritte der oben genannten Themen und das ITA hat nunmehr die Chance, dem RFT und den beiden mit Energietechnologieforschung befassten Ministerien BMVIT und BMWA nachzuweisen, dass mittels eines partizipativen Verfahrens ein interessanter und für die laufende Formulierung dieses Politikbereichs relevanter Beitrag geleistet werden kann.

\section{Future Search \& Assessment „Energie und EndverbraucherInnen"}

Ausgangspunkt des laufenden ITA-Projekts „Future Search \& Assessment - Energie und EndverbraucherInnen“ (kurz: FSA-Energie) bildet der Themenbereich „Energie und Endverbraucher" des Forschungsprogramms „Energie der Zukunft" des BMVIT.

Aktualität und hohes öffentliches Interesse zeichnen diesen Themenbereich besonders für die Durchführung eines Beteiligungsverfahrens und die damit verbundene Bewusstseinsbildung aus. Nach Angaben der österreichischen Energieagentur geben die inländischen Haushalte jährlich rund zehn Mrd. Euro für Energie aus. ${ }^{5}$ Zudem ist der Energieverbrauch der privaten Haushalte in den letzten Jahren stark angestiegen. Die Aktualität des Themas zeigt sich auch in der zunehmend wahrgenommenen Notwendigkeit, den Energieverbrauch drastisch zu senken, um globalen Veränderungsprozessen wie dem Klimawandel entgegenzuwirken.

Im Rahmen einer zweitägigen Veranstaltung am letzten November-Wochenende 2007 haben sich 36 - extra zu diesem Zweck rekrutierte, so weit wie möglich repräsentativ für die österreichische Bevölkerung ausgewählte BürgerInnen mit den Leitbildern auseinandergesetzt, die dem Forschungsprogramm im engeren Sinne und der österreichischen Energieforschung im weiteren Sinne zu Grunde liegen. Diese Leitbilder wurden im Vorfeld durch das ITA-Team identifiziert und in ihre wesentlichen Komponenten zerlegt. Die TeilnehmerInnen haben in einer ersten Phase diese Leitbildelemente diskutiert und kommentiert und zu einem gemeinsamen Leitbild neu zusammengesetzt.

Mit Bezug zur Lebenswelt der TeilnehmerInnen wurden in einer zweiten Phase (am zweiten Tag) die Sozialverträglichkeit ausgewählter Forschungsfragen bzw. die Technologieperspektiven auf dem Weg zur Zielerreichung (mehr Energieeffizienz, weniger Energieverbrauch) diskutiert und bewertet. Der Begriff „Sozialverträglichkeit“ umfasst in diesem Zusammenhang sowohl gemeinwohlorientierte Aspekte (Leitbild) als auch Fragestellungen, die unmittelbar die Aneignung möglicher technischer Anwendungen durch die NutzerInnen (etwa in Form veränderter täglicher Routinen) betreffen.

In einem Vorbereitungsworkshop im September 2007 wurden diese, anschließend im Detail von den BürgerInnen zu behandelnden Themen, vorausgewählt: „Mikro-Kraft-WärmeKopplung“, „Visualisierung und Monitoring des Energieverbrauchs“, „Neue Systemlösungen und Vermeidungsstrategien“" sowie „Innovative Leasing- und Contracting-Modelle“.

Die wichtigsten Ergebnisse der partizipativen Hauptveranstaltung im November wurden im Rahmen einer - nicht vom ITA organisierten - anschließenden ExpertInnenkonferenz den wesentlichen Zielgruppen aus Politik, Verwal- 
tung und Wissenschaft präsentiert. Die BürgerInnen erhielten dadurch auch die Gelegenheit, die Ergebnisse direkt an EntscheidungsträgerInnen und ExpertInnen heranzubringen.

Ziel des Prozesses war somit die Formulierung von Empfehlungen zu den im Zuge des Verfahrens aufgeworfenen Themen, die für die Forschungspolitik sowohl inhaltlich als auch auf legitimatorischer Ebene relevant sind. Darüber hinaus hofft ITA, dass das Ergebnis der Veranstaltung eine wertvolle Grundlage für die Kommunikation mit der Öffentlichkeit darstellen wird. Durch eine gut funktionierende Kommunikation können die potenziellen NutzerInnen frühzeitig mögliche Bedenken, aber auch positive Aspekte und gänzlich neue Wünsche artikulieren. Derartiges Wissen kann dazu verwendet werden, Anforderungen an und Auswahlkriterien für konkrete Forschungsprojekte zu ergänzen.

\section{Ausblick}

Es ist zweifellos noch $\mathrm{zu}$ früh, ein adäquates Fazit zu ziehen, ob die vom ITA ausgegangene Initiative in Richtung verstärkter Einbeziehung von BürgerInnen die Formulierung österreichischer Technologie- und Forschungspolitiken nachhaltig gestalten wird. Dass der Rat für Forschung und Technologieentwicklung jedoch bereits 2003 eine BürgerInnenkonferenz durchführen ließ (damals zum Thema „Genetische Daten"), anschließend das ITA beauftragte, die Perspektive einer verstärkten Einbeziehung der Öffentlichkeit in die Technologiepolitik auszuloten (Projekt Techpol 2.0), und die Ergebnisse nicht nur begrüßt, sondern darüber hinaus die Geschäftsstelle des Rates beauftragt hat, 2007 einen weiteren Pilotversuch zu machen und dazu auch aktiv nach Partnern in den thematisch zuständigen Ministerien zu suchen (Projekt FSAEnergie), stimmt optimistisch. Das ITA ist sich freilich bewusst, dass es unter anderem auch von der gelungenen Vermittlung der Ergebnisse des laufenden Projekts FSA-Energie abhängen wird, ob und wie diese Herangehensweise in Zukunft fortgeführt wird. Die Reaktionen der bei der Präsentation anwesenden Forschungsstaatssekretärin, eines Mitglieds des RFT sowie der VertreterInnen der Verwaltung waren bereits sehr positiv. Wir beobachten weiters auch be- reits in anderen Bereichen eine steigende Aufgeschlossenheit gegenüber einer frühzeitigen Einbeziehung der potenziellen NutzerInnen in die Ausgestaltung von Forschungsprogrammen - so zuletzt im Rahmen des neuen Programms „BeneFIT" des BMVIT, in dem es um die Förderung der Entwicklung von Technologien für ältere Menschen geht.

\section{Anmerkungen}

1) Die neun analysierten Verfahren sind: Zukunftswerkstatt, Future Search Conference / Scenario Workshop, Voting Conference, Dialogverfahren / Runder Tisch, Konsensuskonferenz, Citizen Jury, Planungszelle, Delphi-Verfahren und Fokusgruppen.

2) In diesem Raster unterscheidet das ITA drei wesentliche Dimensionen: den Repräsentationstyp (Einbeziehung von Laien versus ExpertInnen bzw. Stakeholdern), die politische Handlungsform (explorativ versus rahmensetzend) und die Thematisierungsweise (Werte versus Wissen bzw. Interessen). Es würde den Rahmen dieses Beitrags sprengen, die Herleitung dieses heuristischen Instruments näher darzustellen (siehe jedoch Nentwich et al. 2006, insbes. S. 13-21). Das Instrument hat sich nach unserer Auffassung bewährt, um einerseits der Unterschiedlichkeit der typischen partizipativen Verfahren gerecht $\mathrm{zu}$ werden und um andererseits den verschiedenen Charakteristika der untersuchten Technologiethemen zu entsprechen. Aus der Kombination der Zuordnung der Verfahren und der Themen in unserer Matrix ergaben sich wertvolle Hinweise für geeignete Verfahren je Thema.

3) Neben diesen drei Hauptvorschlägen regte das ITA außerdem zwei zusätzliche aktuelle Themen für eine Citizen Jury an: die „Elektronische lebensbegleitende Gesundheitsakte" (ELGA), deren Umsetzung in Österreich ab 2007 geplant wird, und das Thema „Biobanken“, das zuletzt auch die Bioethikkommission im Bundeskanzleramt beschäftigt hat. Abschließend schlagen wir dem RFT und der FFG für die Findung von zukünftigen technologiepolitischen Schwerpunktsetzungen regelmäßige (etwa im Dreijahresrhythmus abgehaltene) Delphiverfahren unter Einbeziehung von ExpertInnen und Stakeholdern vor.

4) Siehe dazu http://www.energiederzukunft.at.

5) 2006 bedeutet dies im Schnitt monatlich $228 €$ pro Haushalt (45\% für Treibstoffe, $33 \%$ für Heizung und Warmwasser und $22 \%$ für Strom) (Quelle: http://www.energyagency.at/(de)/enz/ epi/i-06.htm). 


\section{Literatur}

Nentwich, M.; Bogner, A.; Peissl, W. et al., 2006: Techpol 2.0: Awareness - Partizipation - Legitimität. Vorschläge zur partizipativen Gestaltung der österreichischen Technologiepolitik. Wien: Institut für Technikfolgen-Abschätzung, Endbericht, Studie im Auftrag des RFT, BMWA, BMBWK und BMVIT; http://epub.oeaw.ac.at/ita/ita-projektberichte/d22e15-2.pdf

Steyaert, S.; Lisoir, H.; Nentwich, M. (Hg.), 2006: Leitfaden partizipativer Verfahren. Ein Handbuch für die Praxis. Brüssel, Wien: Flemish Institute for Science and Technology Assessment, König-BaudouinStiftung, Institut für Technikfolgen-Abschätzung; http://epub.oeaw.ac.at/ita/ebooks/Leitfaden_pTA_DE _Feb06.pdf

\section{Kontakt}

PD Dr. Michael Nentwich

Institut für Technikfolgen-Abschätzung Österreichische Akademie der Wissenschaften Strohgasse 45/5, 1030 Wien, Österreich Tel.: +43 (1) 51581 - 6583

E-Mail: mnent@oeaw.ac.at

Internet: http://www.oeaw.ac.at/ita
Bewertung und Kommunikation von chemischen Risiken und kumulierten Stressfaktoren Ein Zwischenbericht zum Projekt "NoMiracle“

\author{
von Christina Benighaus und Ortwin Renn, \\ DIALOGIK Stuttgart
}

Neu eingeführte Technologien führen dazu, dass Mensch und Umwelt neben den natürlichen Stressfaktoren vielfältigen anthropogen verursachten Schadstoffen, Strahlung und Lärm ausgesetzt sind. Die mittel- und langfristigen Wirkungen dieser Expositionen einzeln und in ihrer Kombination sind oftmals nicht geklärt. NoMiracle (NOvel Methods for Integrated Risk Assessment of CumuLative stressors in Europe) ist ein EUVerbundprojekt, das neue Methoden entwickelt, um Risiken von chemischen, physikalischen oder biologischen Substanzen besser abschätzen zu können. Dazu werden die verschiedenen Risiken in ihrer kumulierten Wirkung betrachtet.

Das Projekt NoMiracle ${ }^{1}$ entwickelt einen integrierten Forschungsrahmen zur Beschreibung und Interpretation von so genannten kombinierten Stressfaktoren, die zur Identifikation von Biomarkern and zur Entwicklung von weiteren Indikatoren für die Erfassung von Kombinationseffekten führen und liefert damit ein neues Verständnis für eine kombinierte Risikobewertung. Das Projekt will klären, wie der Austausch von Stoffen zwischen verschiedenen Umweltbestandteilen abläuft und wie diese Prozesse durch natürliche Stressfaktoren beeinflusst werden.

Durch diese Zielsetzung versucht NoMiracle, Mängeln der bisherigen Risikobewertung von chemischen Substanzen entgegenzuwirken. $\mathrm{Zu}$ solchen Mängeln zählen beispielsweise, dass die meisten Verfahren Unsicherheitsfaktoren in Rechnung stellen, die nicht auf wissenschaftlichen Messungen, sondern auf pauschalen Sicherheitsabständen beruhen. 